\title{
AN ASYMPTOTIC THEORY FOR DISPERSION OF REACTIVE CONTAMINANTS IN PARALLEL FLOW
}

\author{
N. G. BARTON
}

(Received 22 March 1982; revised 2 December 1982)

\begin{abstract}
The classic problem, first treated by Taylor [18], of the dispersion of inert soluble matter in fluid flow continues to attract attention from researchers describing the approach to the asymptotic state $[5,17]$. The present article considers some of the complications caused when the solute is chemically active. Dispersing chemically active solutes occur in diverse fields such as chromatography, chemical engineering and environmental fluid mechanics.

The asymptotic large-time analysis of Chatwin [5] is re-worked to handle the case of reactive solutes dispersing in parallel flow. Matching between moderate and large-time solutions requires consideration of the integral moments of the reactive contaminant cloud, and the Aris method of moments is therefore invoked and modified for reaction effects. The results are applied in detail to the outstanding practical example-the chemical flow reactor (a device used to measure reaction rates for chemical reactions taking place between fluids). For this case, the paper provides a practical alternative to recent variable diffusion coefficient studies $[6,7,15]$, and presents further results for the concentration distribution and for the limiting behaviour under weak and vigorous reactions at the boundary of the flow.
\end{abstract}

\section{Introduction}

The dispersion of soluble matter in fluid flow, originally described by G. I. Taylor $[18,19]$, has important applications in a number of fields such as chemical engineering and chromatography [11, 12] and environmental fluid mechanics [9]. Most previous dispersion research has been concerned with short and large time asymptotic theories for passive (neutrally buoyant and chemically inert) contaminants injected into parallel flow. In fact, however, many applications involve

\footnotetext{
'CSIRO Division of Mathematics and Statistics, P. O. Box 218, Lindfield, N.S.W. 2070, Australia. (c) Copyright Australian Mathematical Society 1984, Serial-fee code 0334-2700/84
} 
interesting complications and this paper examines one such case-the dispersion of reactive contaminants in parallel flow. We commence with the mathematical statement of the problem, and then briefly review the progress that has already been made on it.

The object of this paper is to investigate certain aspects of the solution $C(x, y, z, t)$ of the problem

$$
\begin{gathered}
\frac{\partial C}{\partial t}+P u(y, z) \frac{\partial C}{\partial x}=K(y, z) \frac{\partial^{2} C}{\partial x^{2}}+\frac{\partial}{\partial y}\left(K \frac{\partial c}{\partial y}\right) \\
+\frac{\partial}{\partial z}\left(K \frac{\partial C}{\partial z}\right)-\alpha C \text { in } \Omega, \\
\text { n. } K \nabla C=-\beta C \text { at } \partial \Omega, \\
C(x, y, z, 0)=\mathcal{C}(x, y, z), \\
C \text { finite and }\left|x^{m} \frac{\partial^{n} C}{\partial x^{n}}\right| \rightarrow 0 \text { as }|x| \rightarrow \infty \text { for } m, n=0,1,2, \ldots
\end{gathered}
$$

These equations describe the dispersion of a cloud of reactive solute injected into the parallel flow $(u(y, z), 0,0)$ through the cross-sectional region $\Omega$ with boundary $\partial \Omega$. The problem has been written in dimensionless variables, and dimensional variables and constants (denoted by asterisks) may be recovered using the definitions

$$
\begin{gathered}
{\left[x^{*}, y^{*}, z^{*}\right]=a^{*}[x, y, z],} \\
t^{*}=a^{* 2} t / D^{*}, \quad u^{*}=U^{*} u, \\
\kappa^{*}=D^{*} K, \quad C^{*}=Q^{*} c / a^{* 3}|\Omega|, \\
\alpha^{*}=D^{*} \alpha / a^{* 2}, \quad \beta^{*}=\beta / a^{*},
\end{gathered}
$$

where $D^{*}$ and $U^{*}$ are the mean values of $\kappa^{*}$ and $u^{*}$ over $\Omega^{*}, a^{*}$ is a typical cross-sectional length, $Q^{*}$ is the initial amount of solute, and $|\Omega|$ is the dimensionless cross-sectional area of the flow. The dimensionless diffusive flux of contaminant is given by $-K \nabla C$ and $P=U^{*} a^{*} / D^{*}$, the Péclet number of the flow, gives the relative magnitude of typical convection speeds compared to typical cross-sectional diffusive speeds. The inclusion of the factor $|\Omega|$ in the nondimensionalization of $C^{*}$ conveniently ensures that $\iiint C d V=|\Omega|$ initially, and the variables $K$ and $u$ have unit cross-sectional means,

$$
\bar{K}=\frac{1}{|\Omega|} \iint_{\Omega} K(y, z) d y d z=1, \quad \bar{u}=\frac{1}{|\Omega|} \iint_{\Omega} u(y, z) d y d z=1 .
$$

The term $-\alpha C$ on the right hand side of the defining problem represents a first order reaction between the solute and the solvent, whilst the term $-\beta C$ in the defining boundary condition represents a (possibly catalysed) boundary reaction. (Some properties of $\beta$ for laboratory applications are described in reference [8].) 
As a first observation, the effects of the term involving $\alpha$ may be removed immediately by writing $C=C^{\prime} \exp \{-\alpha t\}$, and so we can set $\alpha=0$ without loss of generality. The case when $\beta=0$ as well described the dispersion of a passive contaminant under the usual no diffusive flux boundary condition, and this case is covered by an extensive literature which will be briefly surveyed below. The emphasis in this paper is on the case $\beta \neq 0$.

An initial cloud of inert contaminant $C(x, y, z)$ evolves by cross-sectional diffusion across the velocity shear as it is swept along with the flow. This dispersion process produces a cross-sectional distribution which is, at large times, approximately Gaussian in $x$, and the most important questions to be answered are how much of the cloud remains, where is its centre of mass, and what is the variance and skewness of the distribution? Sophisticated theories attempt to answer these questions for all time, or obtain expressions for the mean concentration $\bar{C}(x, t)$ itself. For the case of no boundary reactions $(\beta=0)$, the dispersion process has been studied extensively since the pioneering work of Taylor $[18,19]$, and results for large times have been obtained by at least four alternative methods: the Taylor-Gill approximation [10], the Aris method of integral moments [1], the Chatwin asymptotic expansion [5], and numerically. A recent paper by Smith [17] (on improvements to the Taylor-Gill approximation) contains further references to most of the important research on the dispersion process. (Smith [16] has also described the early stages of contaminant dispersion, but short-time approximations are not considered in the present article.)

In contrast, the case of $\beta \neq 0$ has received scant attention from theorists, despite the importance of boundary reactions (or models of them) in diverse fields such as biology, physiology, chromatography, chemistry and, of course, in environment fluid mechanics. The most notable work, by Sankarasubramanian and Gill [15] and De Gance and Johns [6,7] has been based on the Taylor-Gill approximation

$$
C(x, y, z, t)=\sum_{n=0}^{\infty} f_{n}(y, z, t) \frac{\partial_{n} \bar{C}}{\partial x^{n}} .
$$

Back substitution of (1.2) into (1.1) ultimately leads to a variable coefficient equation for $\bar{C}$,

$$
\frac{\partial \bar{C}}{\partial t}=K_{0}(t) \bar{C}+K_{1}(t) \frac{\partial \bar{C}}{\partial x}+K_{2}(t) \frac{\partial^{2} \bar{C}}{\partial x^{2}}+\cdots,
$$

which may be truncated and solved. The cited works $[6,7,8,15]$ are mainly concerned with the $\beta$-dependence of the asymptotic forms of $K_{0}(t), K_{1}(t), K_{2}(t)$ at large times. The Taylor-Gill scheme has certain attractive mathematical features as explained by de Gance and Johns, but it is not necessarily the most efficient method at relatively large dimensionless times when a direct large-time 
Chatwin style asymptotic expansion may as well be employed. Therefore, the principal objects of the present article are to present such a direct asymptotic expansion and, in doing so, to extend the results of Sankarasubramanian and Gill [15] for the flow reactor. (The term flow reactor refers to case when the basic flow is laminar flow in a circular pipe; flow reactors are in wide use for determining reaction rates for chemical reactions between mixtures and fluids.)

Matching between the moderate and large-time solutions requires a knowledge of the integral moments of the cloud of reactive solute. Thus, a further goal of the paper is to apply the Aris method of moments to the case with $\beta \neq 0$. The method of moments is known to produce readily the dispersion coefficient for the case $\beta=0$ [1] and for the coated tube chromatograph [2], and useful results prove also to be easily attainable for the case $\beta \neq 0$. To be specific, the technique is shown to give the asymptotic expressions for the amount of solute and its mean position and spread, and it determines arbitrary constants in the asymptotic solution developed in Section 3.

\section{The integral moments of the reactive solute cloud}

Aris [1] first used the moments of an injected solute cloud to describe the dispersion process; in particular, a knowledge of the asymptotic form of the second moment about the mean gives the 'effective diffusion' or 'dispersion' coefficient for the dispersion process. The dispersion coefficient often differs markedly from the ordinary molecular diffusion coefficient: for example, Aris showed from the second moment that the dispersion coefficient is $\kappa^{*}\left(1+P^{2} / 48\right)$ for a solute with constant molecular diffusivity injected into a tube of radius $a^{*}$ containing fluid in laminar flow. Thus the dispersion process is a much more vigorous than normal molecular diffusion for large $P$. In the case of a reactive solute, it is expected that the method of moments will readily determine (at least for large times) how much of the cloud remains, how fast its centre of mass moves, and how much it has spread out. Moreover, as Chatwin [5, Section 6] points out, it is possible to calculate approximations for $\bar{C}(x, t)$ given a knowledge of the first three or four integral moments.

The approach adopted in this section follows that recently used by the author [3] in calculating the moments for passive contaminants. Thus, the moments $C_{n}(y, z, t)$ and $M_{n}(t)$ are defined by

$$
\begin{gathered}
C_{n}(y, z, t)=\int_{-\infty}^{\infty} x^{n} C(x, y, z, t) d x, \\
M_{n}(t)=\bar{C}_{n}=\frac{1}{|\Omega|} \iint_{\Omega} C_{n}(y, z, t) d y d z,
\end{gathered}
$$


and it may be readily established that $C_{n}$ and $M_{n}$ are the solutions of the problems

$$
\begin{gathered}
\frac{\partial C_{n}}{\partial t}-\frac{\partial}{\partial y}\left(K \frac{\partial C_{n}}{\partial y}\right)-\frac{\partial}{\partial z}\left(K \frac{\partial C_{n}}{\partial z}\right)=n P u C_{n-1}+n(n-1) K C_{n-2}, \\
C_{n}(y, z, 0)=\mathcal{C}_{n}(y, z), \\
K \nabla C_{n} \cdot \hat{\mathbf{n}}=-\beta C_{n} \quad \text { at } \partial \Omega
\end{gathered}
$$

and

$$
\begin{gathered}
\frac{d M_{n}}{d t}=-\frac{1}{|\Omega|} \oint_{\partial \Omega} \beta C_{n} d s+n P \overline{u C_{n-1}}+n(n-1) \overline{K C_{n-2}}, \\
M_{n}(0)=\overline{\mathcal{C}}_{n}=\Re_{n}
\end{gathered}
$$

where the overbar denotes the cross-sectional mean.

The first term on the right hand side of $(2.4 \mathrm{a})$ is caused by the reaction at the boundary of the flow, and implies that a knowledge of $C_{n}$ is required before using (2.4a) to calculate $M_{n}$. This is in contrast to the case of passive contaminants in which $\beta=0$ and one needs $C_{n-1}$ and $C_{n-2}$, but not $C_{n}$, to calculate $M_{n}$. Thus, for $\beta \neq 0$, there is nothing to be gained by using equations (2.4); instead, solutions for $C_{n}$ (up to $n=2$ ) will be obtained by separating variables in equations (2.3) and $M_{n}(t)$ follows by taking the cross-sectional mean.

For $n=0$, the solution to (2.3) may be written

$$
C_{0}(t)=\sum_{i} A_{0 i} f_{i} e^{-\mu, t}
$$

where it is assumed that $K(y, z)$ is such that the eigenvalue problem

$$
\begin{gathered}
L_{i}\left(f_{i}\right)=\left\{\frac{\partial}{\partial y}\left(K \frac{\partial}{\partial y}\right)+\frac{\partial}{\partial z}\left(K \frac{\partial}{\partial z}\right)+\mu_{i}\right\} f_{i}=0, \\
K \nabla f_{i} \cdot \hat{\mathbf{n}}=-\beta f_{i} \quad \text { at } \partial \Omega, \quad f_{i} \text { finite, }
\end{gathered}
$$

possesses a discrete spectrum of real eigenvalues $\left\{\mu_{1}\right\}_{i=1}^{\infty}$ ordered so that $0<\mu_{1}<$ $\mu_{2}<\mu_{3}<\cdots$ and a corresponding complete set of eigenfunctions that are orthogonal and normalized so that

$$
\overline{f_{i} f_{j}}= \begin{cases}1, & i=j \\ 0, & i \neq j\end{cases}
$$

Fitting the initial condition $C_{0}(y, z, 0)=\bigodot_{0}(y, z)$ determines the constants $A_{0 i}$ in (2.5) to be

$$
A_{0}=\overline{\mathcal{C}_{0} f_{\imath}}
$$

and so we have

$$
C_{0}(y, z, t,)=\sum_{t} \overline{\mathcal{C}_{0} f_{i}} f_{i} e^{-\mu, t}
$$


The problem for $C_{1}$ is more complicated, however, since certain solvability conditions have to be satisfied to obtain the solution. A discussion of these solvability conditions has recently been given by the author [3] for the case with $\beta=0$, and the methods required for the present problem follow naturally from those in the reference. Thus the defining equation for $C_{1}(y, z, t)$ is modified by adding in and subtracting out inhomogeneous terms chosen so that the problem for $C_{1}$ is solvable. That is, we write

$$
\begin{aligned}
\frac{\partial C_{1}}{\partial t}-\frac{\partial}{\partial y}\left(K \frac{\partial C_{1}}{\partial y}\right)-\frac{\partial}{\partial z}\left(K \frac{\partial C_{1}}{\partial z}\right) & =P u C_{0}=P \sum_{t} A_{0,} u f_{\imath} e^{-\mu_{t} t} \\
& =P \sum_{t} A_{0 i}\left(u-\gamma_{\imath}\right) f_{i} e^{-\mu_{t} t}+P \sum_{i} A_{0,} \gamma_{l} f_{t} e^{-\mu_{t} t},
\end{aligned}
$$

where the solution of this equation under the boundary condition $(2.3 \mathrm{c})$ is

$$
C_{1}=\sum_{i}\left\{A_{1 \imath} f_{t}+P A_{0 \iota}\left(\phi_{t}+t \gamma_{t} f_{\imath}\right)\right\} e^{-\mu_{\imath} t} .
$$

Here, the particular solutions $\phi_{t}$ are the solutions of

$$
\begin{gathered}
L_{\imath} \phi_{l}=-\left(u-\gamma_{\imath}\right) f_{i}, \\
K \nabla \phi_{l} \cdot \hat{\mathbf{n}}=-\beta \phi_{l} \quad \text { at } \partial \Omega, \quad \phi_{l} \text { finite, }
\end{gathered}
$$

and, as is well-known from eigenvalue theory, the solution $\phi_{l}$ exists only if the inhomogeneous term $\left(u-\gamma_{t}\right) f_{i}$ in (2.10a) is orthogonal to the eigenfunction $f_{i}$ of the homogeneous problem. The constants $\gamma_{t}$ are therefore found to be

$$
\gamma_{t}=\overline{u f_{t} f_{t}} \text {. }
$$

It is now possible to express $\phi_{t}$ in terms of the complete set of eigenfunctions $\left\{f_{t}\right\}_{i=1}^{\infty}$, that is,

$$
\phi_{t}=\sum_{j} a_{i j} f_{j}
$$

where the coefficients are given by expanding the inhomogeneous terms in (2.10a) in terms of the $\left\{f_{i}\right\}$. After simplification, it is found that

$$
a_{i j}=\left\{\begin{array}{ll}
\overline{u f_{i} f_{j}} /\left(\mu_{j}-\mu_{t}\right), & j \neq i, \\
\text { arbitrary, } & j=1,
\end{array}\right\}
$$

where the $a_{11}$ are hereinafter set equal to zero. The constants $A_{1}$ in (2.9) are then found to be

$$
A_{1 t}=\overline{e_{1} f_{i}}-P \sum_{j \neq l} \overline{e_{0} f_{j}} \overline{u f_{i} f_{j}} /\left(\mu_{t}-\mu_{\jmath}\right)
$$


by fitting the initial condition $C_{1}(y, z, 0)=\mathcal{C}_{1}(y, z)$, and, after simplification, the full solution for $C_{1}$ is found to be

$$
\begin{aligned}
& C_{1}=\sum_{i} \overline{\mathcal{C}_{1} f_{i}} f_{t} e^{-\mu, t}+P \sum_{i} \sum_{j \neq l} \frac{\overline{u f_{t} f_{j}}}{\left(\mu_{j}-\mu_{t}\right)}\left\{\overline{\mathcal{C}_{0} f_{i}} f_{j}+\overline{\bigodot_{0} f_{j}} f_{i}\right\} e^{-\mu_{t} t} \\
& +P t \sum_{i} \overline{\mathcal{C}_{0} f_{i}} \overline{u f_{i} f_{i}} f_{i} e .
\end{aligned}
$$

The moment $C_{2}$ is obtained by solving the problem (2.3) with $n=2$, and the analysis becomes quite laborious at this point. To solve (2.3) with $n=2$, various terms have to be added into and taken out of the inhomogeneous terms to ensure solvability, and, omitting the tedious details, $C_{2}$ is found to be

$$
C_{2}=\sum_{l}\left\{A_{2 l} f_{l}+\psi_{t}+t \delta_{l} f_{l}+P^{2} t A_{0 l} \gamma_{l}\left[2 \phi_{l}+t \gamma_{l} f_{l}\right]\right\} e^{-\mu_{l} t}
$$

where $\psi_{l}$ is the particular solution of

$$
-L_{t} \psi_{t}=2 A_{0 i} K f_{i}+2 P\left[A_{1} u f_{i}+P A_{0 i}\left(u-\gamma_{t}\right) \phi_{t}\right]-\delta_{t} f_{t}
$$

under the boundary conditions (2.3c). The constants $\delta_{\text {, }}$ are found to be

$$
\delta_{i}=2 A_{0 i} \overline{K f_{i} f_{i}}+2 P\left[A_{1} \overline{u f_{i} f_{i}}+P A_{0 i} \overline{\left(u-\gamma_{t}\right) \phi_{i} f_{i}}\right]
$$

by applying the usual solvability condition. Also, the $\psi_{i}$ can be expressed in the form

$$
\psi_{i}=\sum_{j} b_{i j} f_{j}
$$

where the $b_{i j}$ are ultimately found to be

$$
b_{\imath j}=\left\{\begin{array}{ll}
\left\{2 A_{0} \overline{K f_{\imath} f_{j}}+2 P\left[A_{l} \overline{u f_{\imath} f_{j}}+P A_{0} \overline{\left(u-\gamma_{l}\right) \phi_{\imath} f_{j}}\right]\right\} /\left(\mu_{j}-\mu_{i}\right), & j \neq i, \\
\text { arbitrary, } & j=i .
\end{array}\right\}
$$

If the coefficients $b_{t}$, are also set equal to zero, $C_{2}$ is completely specified by assigning the constants $A_{2 i}$ to fit the initial condition $C_{2}(y, z, 0)=e_{2}(y, z)$. The procedure is again straightforward and gives

$$
A_{2 i}=\overline{\mathcal{C}_{2} f_{i}}-\sum_{\jmath} b_{j l} \text {. }
$$

The foregoing mathematics has therefore determined $C_{0}, C_{1}$ and $C_{2}$ and could be used to determine three-term approximations to $\bar{C}(x, t)$ at any time by following the procedures described by Chatwin [5, Section 6]. To do this, the first few moments about the mean are required; they are defined by

$$
\nu_{n}(t)=\frac{\iiint(x-\bar{x})^{n} C d V}{\iiint C d v}
$$


where

$$
\bar{x}=\frac{\iiint x C d V}{\iiint C d V}=\bar{C}_{1} / \bar{C}_{0}
$$

and it follows that

$$
\begin{gathered}
\nu_{2}(t)=\bar{C}_{2} / \bar{C}_{0}-\bar{x}^{2}, \\
\nu_{3}(t)=\left(\bar{C}_{-3} / \bar{C}_{\hat{v}}\right) 3 \bar{x}_{2}-\bar{x}^{3} .
\end{gathered}
$$

Of course, a lot of numerical work is required to calculate the eigenvalues and eigenfunctions and then the $\bar{C}_{n}(t)$ for general values of time. A calculation of $\bar{C}_{3}$ would give a four-term approximation to $\bar{C}(x, t)$, and, in view of the complexity of $C_{2}$ that has already been shown, it would appear that $\bar{C}_{3}$ would be the highest moment that is practically obtainable.

The calculation of $\bar{C}_{0}(t), \bar{C}_{1}(t)$ and $\bar{C}_{2}(t)$ does, however, enable some simple and interesting conclusions to be drawn for asymptotically large times. If the notation EST is used to denote exponentially small terms, the largest of which is of order $\exp \left\{-\left(\mu_{2}-\mu_{1}\right) t\right\}$, the results above can be used to establish that, for large $t$,

$$
\begin{gathered}
\bar{C}_{0}=A_{0 t} \bar{f}_{1} e^{-\mu_{1} t}(1+E S T), \\
\bar{x}=\gamma_{1} P t+\left(A_{11} / A_{01}+P \bar{\phi}_{1} / \bar{f}_{1}\right)+E S T,
\end{gathered}
$$

and

$$
\begin{aligned}
\nu_{2}(t)= & 2\left\{\overline{K f_{1} f_{1}}+P^{2} \overline{\left(u-\gamma_{1}\right) \phi_{1} f_{1}}\right\} t \\
& +\frac{A_{21}}{A_{01}}+\frac{\bar{\psi}_{1}}{A_{01} \bar{f}_{1}}-\left(\frac{A_{11}}{A_{01}}+\frac{P \bar{\phi}}{\bar{f}_{1}}\right)^{2}+E S T .
\end{aligned}
$$

These expressions (2.20-2.22) therefore describe, for large values of time, the most important features of the reacting solute cloud: the total amount of solute is exponentially decaying and is given by (2.20), its centre of mass moves at the speed $\gamma_{1} P$, and $\bar{C}(x, t)$ ultimately tends to a Gaussian distribution in $x$ with dispersion coefficient $\left\{\overline{K f_{1} f_{1}}+P^{2} \overline{\left(u-\gamma_{1}\right) \phi_{1} f_{1}}\right\}$.

\section{A direct asymptotic expansion for large $t$}

We now consider the problem of finding a direct asymptotic expansion of the solution of equations (1) with $\alpha=0$ and $\beta \neq 0$. The method adopted is a generalization of that used by Chatwin [5] to investigate the asymptotic behaviour of $C$ when wall reactions are absent $(\beta=0)$, and it may be regarded as an 
alternative at large times to the Taylor-Gill approach of references $[6,7,15]$.

Accordingly, the variables $X, Y, Z$ and $T$ defined by

$$
X=(x-\gamma P t) / P T, \quad Y=y, \quad Z=z, \quad T=(M t)^{1 / 2}
$$

are introduced, where $\gamma$ and $M$ are dimensionless constants whose values will be determined shortly. In terms of these variables, the problem (1.1) for $C$ becomes

$$
\frac{M}{2 T} \frac{\partial C}{\partial t}+\left(\frac{u-\gamma}{T}-\frac{X M}{2 T^{2}}\right) \frac{\partial C}{\partial X}=\frac{K}{P^{2} T^{2}} \frac{\partial^{2} C}{\partial X^{2}}+\frac{\partial}{\partial Y}\left(K \frac{\partial C}{\partial Y}\right)+\frac{\partial}{\partial Z}\left(K \frac{\partial C}{\partial Z}\right),
$$

$C$ finite,

$$
\text { î. } K \nabla C=-\beta C \text { at } \partial \Omega,
$$

together with a matching condition between the asymptotic and moderate time solutions. Guided by Chatwin's work and the interpretation of the results of the previous section at large times, an asymptotic solution for $C$ is sought in the form

$$
\begin{aligned}
C=e^{-\mu_{1} T^{2} / M}\left\{C^{(1)}(X, Y, Z) / T+C^{(2)}\right. & (X, Y, Z) / T^{2} \\
& \left.+C^{(3)}(X, Y, Z) / T^{3}+\cdots\right\}
\end{aligned}
$$

where $\mu_{1}$ is the smallest eigenvalue of problem (2.6). The coefficient functions $C_{n}$ in (3.3) satisfy the conditions (3.2b) and the following equations obtained by comparing coefficients of $T^{-n}$ in (3.2a):

$$
\begin{gathered}
L_{1}\left(C^{(1)}\right)=0, \\
L_{1}\left(C^{(2)}\right)=(u-\gamma) \frac{\partial C^{(1)}}{\partial X}, \\
L_{1}\left(C^{(3)}\right)=(u-\gamma) \frac{\partial C^{(n-1)}}{\partial X} \\
-\frac{M}{2}\left\{\frac{2 K}{P^{2} M} \frac{\partial^{2} C^{(n-2)}}{\partial X^{2}}+X \frac{\partial C^{(n-2)}}{\partial X}+(n-2) C^{(n-2)}\right\}, \\
n=3,4, \ldots .
\end{gathered}
$$

Here the operator $L_{1}$ is defined by equation (2.6a) with $i=1$.

The solution of equation (3.4) satisfying conditions (3.2b) is

$$
C^{(1)}(X, Y, Z)=f_{1}(Y, Z) h_{1}(X)
$$

where $h_{1}(X)$ is arbitrary and $f_{1}$ is the eigenfunction corresponding to $\mu_{1}$. Equation (3.5) then has a solution satisfying the boundary condition (3.2b) provided that the solvability condition

$$
\gamma=\overline{u f_{1} f_{1}}
$$


is satisfied. This ensures that the inhomogeneous term in (3.5) is orthogonal to the eigenfunction of the homogeneous equation, and permits the solution of (3.5) to be written

$$
C^{(2)}(X, Y, Z)=f_{1}(Y, Z) h_{2}(X)+g_{2}(Y, Z) \frac{d h_{1}}{d X}
$$

where $h_{2}(X)$ is arbitrary and $g_{2}$ satisfies

$$
i_{1}\left(g_{2}\right)=(u-\gamma) \hat{f_{1}}
$$

under conditions (3.2b). It is noted that, apart from an arbitrary multiple of $f_{1}, g_{2}$ is the negative of $\phi_{1}$ in the previous section. For the rest of this section, it proves convenient to choose this arbitrary multiple of $f_{1}$ so that $g_{2}$ satisfies the auxiliary condition $\bar{g}_{2}=0$.

A differential equation for the arbitrary function $h_{1}(X)$ in (3.7) is obtained by considering the solvability condition which results when (3.7-3.9) are substituted into (3.6) with $n=3$. The right hand side of (3.6) becomes

$$
(u-\gamma) f_{1} h_{2}^{\prime}-\frac{M}{2}\left\{\frac{2}{M}\left[\frac{K f_{1}}{P^{2}}-(u-\gamma) g_{2}\right] h_{1}^{\prime \prime}+X f_{1} h_{1}^{\prime}+f_{1} h_{1}\right\}
$$

and solvability requires that $\gamma=\overline{u f_{1} f_{1}}$ (already known) and

$$
\frac{2}{M}\left[\frac{\overline{K f_{1} f_{1}}}{P^{2}}-\overline{(u-\gamma) g_{2} f_{1}}\right] h_{1}^{\prime \prime}+X h_{1}^{\prime}+h_{1}=0 .
$$

Thus the most convenient description of the solution will be when $M$ takes the value

$$
M=2\left[\overline{K f_{1} f_{1}} / P^{2}-\overline{(u-\gamma) g_{2} f_{1}}\right]
$$

and $h_{1}(X)$ is then

$$
h_{1}(X)=\exp \left\{-\frac{1}{2} X^{2}\right\} .
$$

To continue the construction of the solution, the results $(3.11,3.12)$ are substituted into (3.6) with $n=3$ to obtain

$$
\begin{aligned}
L_{1}\left(C^{(3)}\right)= & (u-\gamma) f_{1} h_{2}^{\prime} \\
& -\left[\frac{1}{P^{2}}\left(K-\overline{K f_{1} f_{1}}\right) f_{1}-\left\{(u-\gamma) g_{2}-\overline{(u-\gamma) g_{2} f_{1}} f_{1}\right\}\right] h_{1}^{\prime \prime} .
\end{aligned}
$$

The solution for $C^{(3)}$ is

$$
C^{(3)}(X, Y, Z)=f_{1}(Y, Z) h_{3}(X)+g_{2}(Y, Z) \frac{d h_{2}}{d X}+g_{3}(X, Z) \frac{d^{2} h_{1}}{d X^{2}}
$$

where $h_{3}(X)$ is arbitrary and $g_{3}(Y, Z)$ is the solution of

$$
L_{1}\left(g_{3}\right)=(u-\gamma) g_{2}-\overline{(u-\gamma) g_{2} f_{1}} f_{1}-\frac{1}{P^{2}}\left(K-\overline{K f_{1} f_{1}}\right) f_{1}
$$


under the conditions (3.2b). The function $g_{3}$ is completely specified by making the further (convenient) restriction that $\bar{g}_{3}=0$.

The pattern of the asymptotic expansion is now clear and the details are quite similar to those given by Chatwin for the case with $\beta=0$. The asymptotic solution involves Hermite polynomials, $H_{n}(X)$, defined by

$$
H_{n}(X) \exp \left\{-\frac{1}{2} X^{2}\right\}=\left(-\frac{d}{d x}\right)^{n} \exp \left\{-\frac{1}{2} X^{2}\right\}
$$

and, in terms of these, the coefficient functions $C^{(1)}, C^{(2)}$ and $C^{(3)}$ are

$$
\begin{gathered}
C^{(1)}=\alpha_{1,0} f_{1} H_{0} \exp \left\{-\frac{1}{2} X^{2}\right\}, \\
C^{(2)}=\left[\left(\alpha_{2,0} f_{1}-\alpha_{1,0} g_{2}\right) H_{1}+\alpha_{2,1} f_{1} H_{3}\right] \exp \left\{-\frac{1}{2} X^{2}\right\} \\
C^{(3)}=\left[\left(\alpha_{3,0} f_{1}-\alpha_{2,0} g_{3}+\alpha_{1,0} g_{3}\right) H_{2}\right. \\
\left.+\left(\alpha_{3,1} f_{1}-\alpha_{2,1} g_{2}\right) H_{4}+\alpha_{3,2} f_{1} H_{6}\right] \exp \left\{-\frac{1}{2} X^{2}\right\}
\end{gathered}
$$

In these expressions, $\alpha_{1,0}, \alpha_{2,0}$ and $\alpha_{3,0}$ are arbitrary constants, $g_{2}$ and $g_{3}$ are the solutions of $(3.10,3.15)$, and the remaining constants are specified by enforcing the solvability conditions as described above. Omitting the tedious details, these constants are found to be

$$
\begin{aligned}
\alpha_{2,1}= & \alpha_{1,0}\left\{\left[\overline{(u-\gamma) g_{3} f_{1}}-\overline{K g_{2} f_{1}} / P^{2}\right] / M+\frac{1}{2} \overline{g_{2} f_{1}}\right\}, \\
\alpha_{3,1}= & -\alpha_{1,0}\left\{\left[\overline{(u-\gamma) g_{4} f_{1}}-\overline{K g_{3} f_{1}} / P^{2}\right] / M+\frac{1}{2} \overline{g_{3} f_{1}}\right\} \\
& +\left(\alpha_{1,0} \overline{g_{2} f_{1}}+\alpha_{2,0}\right)\left\{\left[\overline{(u-\gamma) g_{3} f_{1}}-\overline{K g_{2} f_{1}} / P^{2}\right] / M+\frac{1}{2} \overline{g_{2} f_{1}}\right\}, \\
\alpha_{3,2}= & \frac{1}{2} \alpha_{1,0}\left\{\left[\overline{(u-\gamma) g_{3} f_{1}}-\overline{K g_{2} f_{1}} / P^{2}\right] / M+\frac{1}{2} \overline{g_{2} f_{1}}\right\}^{2} .
\end{aligned}
$$

It now remains to determine the arbitrary constants $\alpha_{n, 0}(n=1,2,3, \ldots)$. These constants depend on the initial distribution and they can be determined using the integral moments (2.19) which were investigated in the previous section. The plan is to determine the moments by integrations of the series (3.3), and, for this purpose, the following properties of the Hermite polynomials are required:

$$
\begin{gathered}
\int_{-\infty}^{\infty} H_{m} H_{n} \exp \left(-\frac{1}{2} X^{2}\right) d X=(2 \pi)^{1 / 2} m ! \delta_{m n}, \\
\int_{-\infty}^{\infty} X^{m} H_{n} \exp \left(-\frac{1}{2} X^{2}\right) d X=\left\{\begin{array}{rr}
0 & \text { if } m<n \text { or }(m-n) \text { is odd }, \\
(2 \pi)^{1 / 2} m !(m-n-1)(m-n-3) \cdots & 1 /(m-n) ! \quad \text { if }(m-n) \text { is even } .
\end{array}\right.
\end{gathered}
$$


Thus, using expressions $(3.3,3.17-3.19)$, we find that

$$
\begin{gathered}
\iiint C d V=P \pi(2 \pi)^{1 / 2} \alpha_{1,0} \bar{f}_{1} e^{-\mu_{1} t}, \\
\bar{x}=\gamma P t+P \alpha_{2,0} / \alpha_{1,0}, \\
\nu_{2}(t) \approx P^{2} M T+2 P^{2} \alpha_{3,0} / \alpha_{1,0}, \\
\eta_{3}(t) \approx 6 P^{3}\left(\tilde{\sim}_{2,1} M t+\hat{u}_{4,0}\right) / \hat{u}_{1,0} .
\end{gathered}
$$

In equation (3.22), it is convenient (as Chatwin points out) to choose the axes of the coordinate system so that $\bar{x}=\gamma P t$ asymptotically. With such a choice, $\alpha_{2,0}$ becomes zero and this simplification has been incorporated in $(3.23,3.24)$.

The calculation of the asymptotic form of the integral moments from the Aris moment equations therefore fixes the constants $\alpha_{n, 0}(n \geqslant 3)$. In particular, using expressions $(2.22,3.23), \alpha_{3,0} / \alpha_{1,0}$ is given by

$$
\frac{\alpha_{3,0}}{\alpha_{1,0}}=\frac{1}{2 P^{2}}\left\{\frac{A_{21}}{A_{01}}+\frac{\bar{\psi}}{A_{01} \bar{f}_{1}}-\left(\frac{A_{11}}{A_{01}}+\frac{P \bar{\phi}_{1}}{\bar{f}_{1}}\right)^{2}\right\}
$$

This expression depends on the initial distribution through the constants $A_{01}, A_{11}$, $A_{21}$ and, in contrast with Chatwin's analogous expression (3.10), further simplification of it is not warranted for $\beta \neq 0$.

Similar procedures would suffice to determine $\alpha_{4,0}$ and the $\alpha_{n, 0}$ for $n \geqslant 5$. These details are not included because they are not required for a three term asymptotic series for $C$ which, as Chatwin points out, is sufficient for most purposes.

\section{Application of the results to the flow reactor}

Perhaps the most important application for the preceding work is to the flow reactor-a device in wide use for measuring reaction rates for chemical reactions taking place in a mixture of fluids $[4,11,12,13,20]$. The flow reactor consists of a circular pipe of radius $a^{*}$ containing a solvent in laminar flow so that $u$ is the Hagen-Poiseuille profile

$$
u(\rho)=2\left(1-\rho^{2}\right), \quad 0 \leqslant \rho \leqslant 1,
$$

where $\rho$ is the usual cylindrical polar coordinate. It will be assumed that the problem is independent of the other polar coordinate $\phi$ and that the diffusion coefficient is constant so that $K=1$; the eigenvalue problem (2.6) then becomes

$$
\left\{\frac{1}{\rho} \frac{d}{d \rho}\left(\rho \frac{d}{d \rho}\right)+\mu_{1}\right\} f_{i}=0,
$$




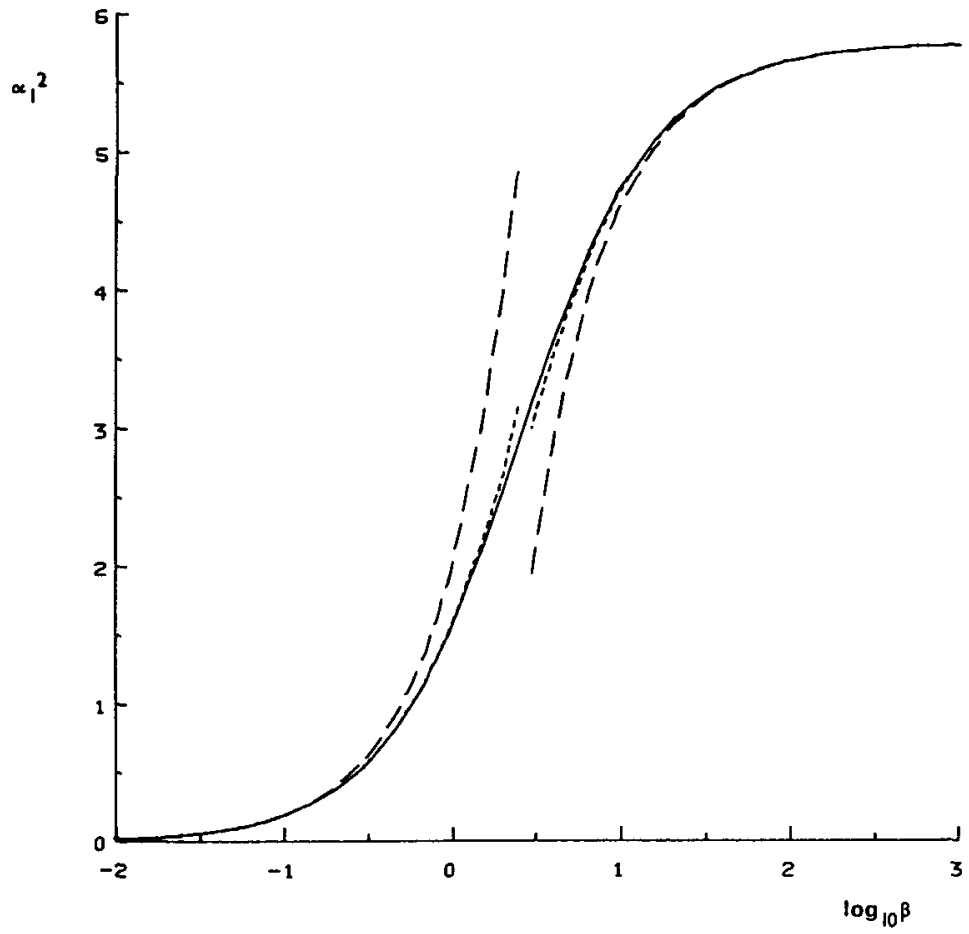

Figure 1. Plots of the parameter $\alpha_{1}^{2}$ against the dimensionless wall reaction parameter $\beta$ :

result of numerical root finding procedures; -----, approximations due to Sankarasubramanian and Gill; ......, approximations developed in the present paper.

$$
\frac{d f_{l}}{d \rho}=-\beta f_{1} \text { at } \rho=1, \quad f_{l} \text { finite }
$$

The eigenvalues and normalized eigenfunctions for the problem are

$$
\psi_{1}=\alpha_{\imath}^{2}, \quad f_{i}(\rho)=\frac{\alpha_{i} J_{0}\left(\alpha_{\imath} \rho\right)}{\left(\alpha_{\imath}^{2}+\beta^{2}\right)^{1 / 2} J_{0}\left(\alpha_{t}\right)}
$$

where $J_{0}$ is the Bessel function of order 0 and $\left\{\alpha_{i}\right\}_{i=1}^{\infty}$ are the roots of

$$
\alpha_{i} J_{0}^{\prime}\left(\alpha_{\imath}\right)=-\beta J_{0}\left(\alpha_{i}\right)
$$

The evaluation of various constants in Sections 2 and 3 requires the standard integrals

$$
\int_{0}^{1} \rho J_{0}\left(\alpha_{\imath} \rho\right) J_{0}\left(\alpha_{j} \rho\right) d \rho= \begin{cases}\frac{1}{2} \alpha_{i}^{-2}\left(\alpha_{i}^{2}+\beta^{2}\right) J_{0}^{2}\left(\alpha_{i}\right), & i=j \\ 0, & i \neq j\end{cases}
$$




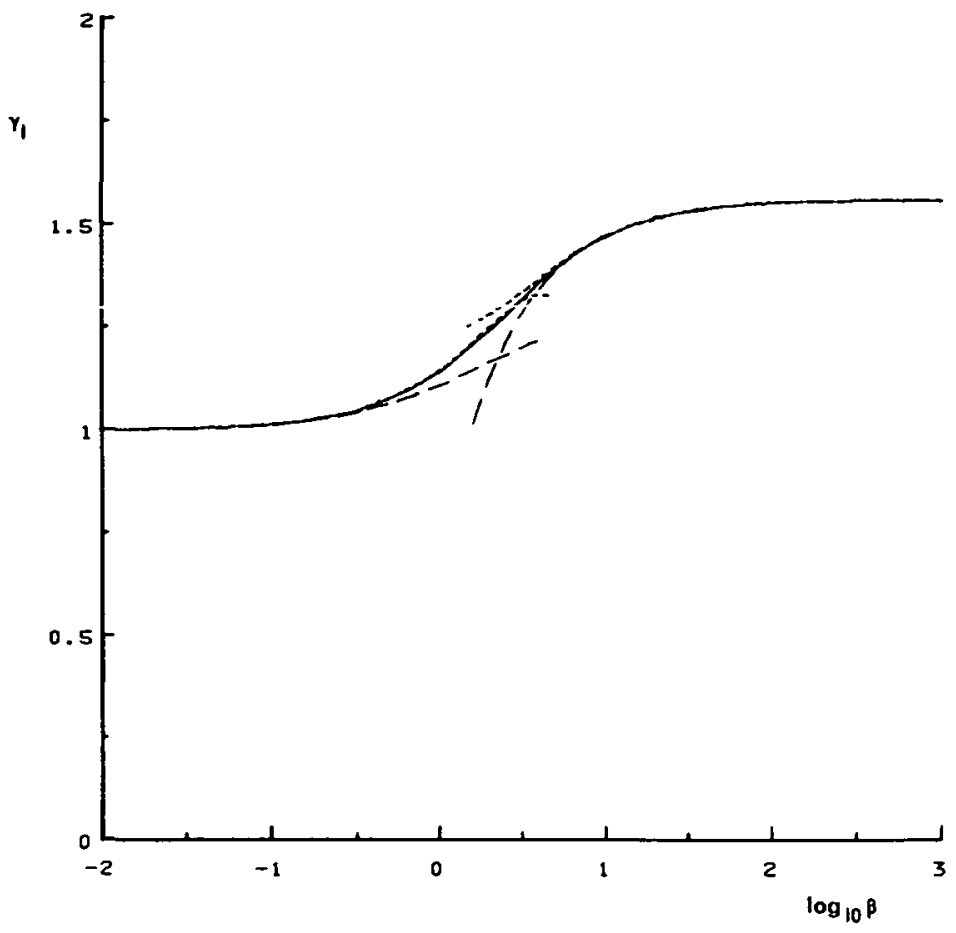

Figure 2. Plots of the dimensionless parameter $\gamma_{1}$ (occurring in $\bar{x} \sim \gamma P t$ ) against the dimensionless wall reaction parameter $\beta$ : , exact expression (4.7); ------, approximations due to Sankarasubramanian and Gill; ....., approximations developed in the present paper.

$$
\int_{0}^{1} \rho^{3} J_{0}\left(\alpha_{i} \rho\right) J_{0}\left(\alpha_{j} \rho\right) d \rho= \begin{cases}\frac{\left[\alpha_{i}^{4}+\alpha_{i}^{2}\left(2 \beta+\beta^{2}\right)-2 \beta^{2}\right] J_{0}^{2}\left(\alpha_{i}\right)}{6 \alpha_{i}^{4}}, & i=j, \\ \frac{2\left(\alpha_{i}^{2}+\alpha_{j}^{2}+2 \beta^{2}\right) J_{0}\left(\alpha_{i}\right) J_{0}\left(\alpha_{\jmath}\right)}{\left(\alpha_{i}-\alpha_{\jmath}\right)^{2}}, & i \neq j,\end{cases}
$$

and the computation of the $\left\{\alpha_{t}(\beta)\right\}_{i=1}^{\infty}$ using standard root-finding procedures. The root $\alpha_{1}(\beta)$ is of particular importance since the total amount of contaminant ultimately decays as $\exp \left\{-\alpha_{1}^{2} t\right\}$. The value of $\alpha_{1}^{2}$ as a function of $\beta$ is displayed in Figure 1 , together with the small and large $\beta$ approximations due to Sankarasubramanian and Gill:

$$
\begin{gathered}
\alpha_{1}^{2} \sim 2 \beta \quad \text { for small } \beta, \\
\alpha_{1}^{2} \sim j_{0,1}^{2}(1-2 / \beta) \quad \text { for large } \beta,
\end{gathered}
$$




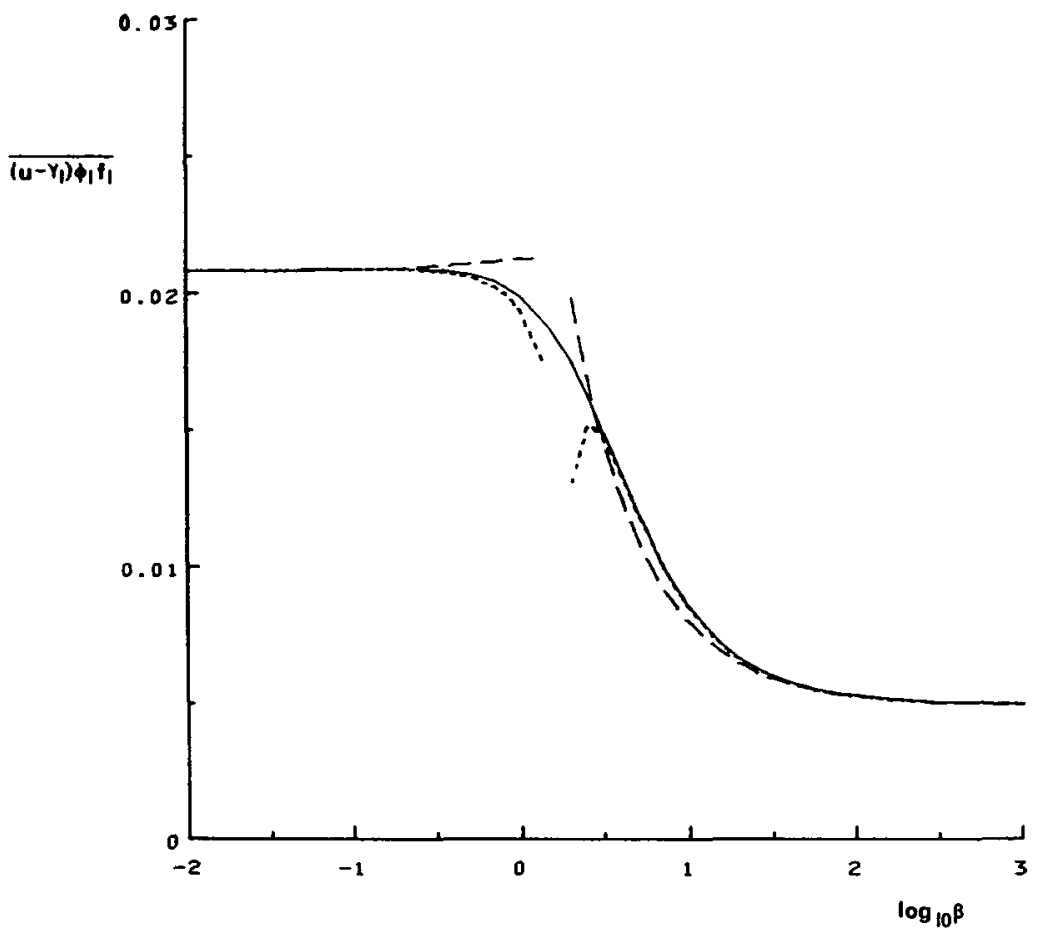

Figure 3. Plots of the dimensionless quantity $\overline{\left(u-\gamma_{1}\right) \phi_{1} f_{1}}$ (occurring in $D_{e f f}=1+P^{2} \overline{\left.\left(u-\gamma_{1}\right) \phi_{1} f_{1}\right)}$ against the dimensionless wall reaction parameter $\beta$ : __ exact expression (4.11) (or by summing (2.12a) and integrating); -----, approximations due to Sankarasubramanian and Gill; ....., approximations developed in the present paper.

where $j_{0,1} \cong 2.40483$ is the first zero of $J_{0}(x)$. Figure 1 also shows the improved small and large $\beta$ approximations

$$
\begin{gathered}
\alpha_{1}^{2} \sim 2 \beta-\frac{1}{2} \beta^{2}+\frac{1}{12} \beta^{3} \quad \text { for small } \beta, \\
\alpha_{1}^{2} \sim j_{0,1}^{2}\left(1-1 / \beta+1 /\left(2 \beta^{2}\right)\right)^{2} \text { for large } \beta,
\end{gathered}
$$

which can be derived without much additional effort.

The centre of mass of the contaminant cloud ultimately moves at the speed $\gamma_{i} P$ where $\gamma_{1}=\overline{u f_{1} f_{1}}$ is found to be

$$
\gamma_{1}=\frac{4\left[\alpha_{1}^{4}+\alpha_{1}^{2}\left(-\beta+\beta^{2}\right)+\beta^{2}\right]}{3 \alpha_{1}^{2}\left(\alpha_{1}^{2}+\beta^{2}\right)}
$$




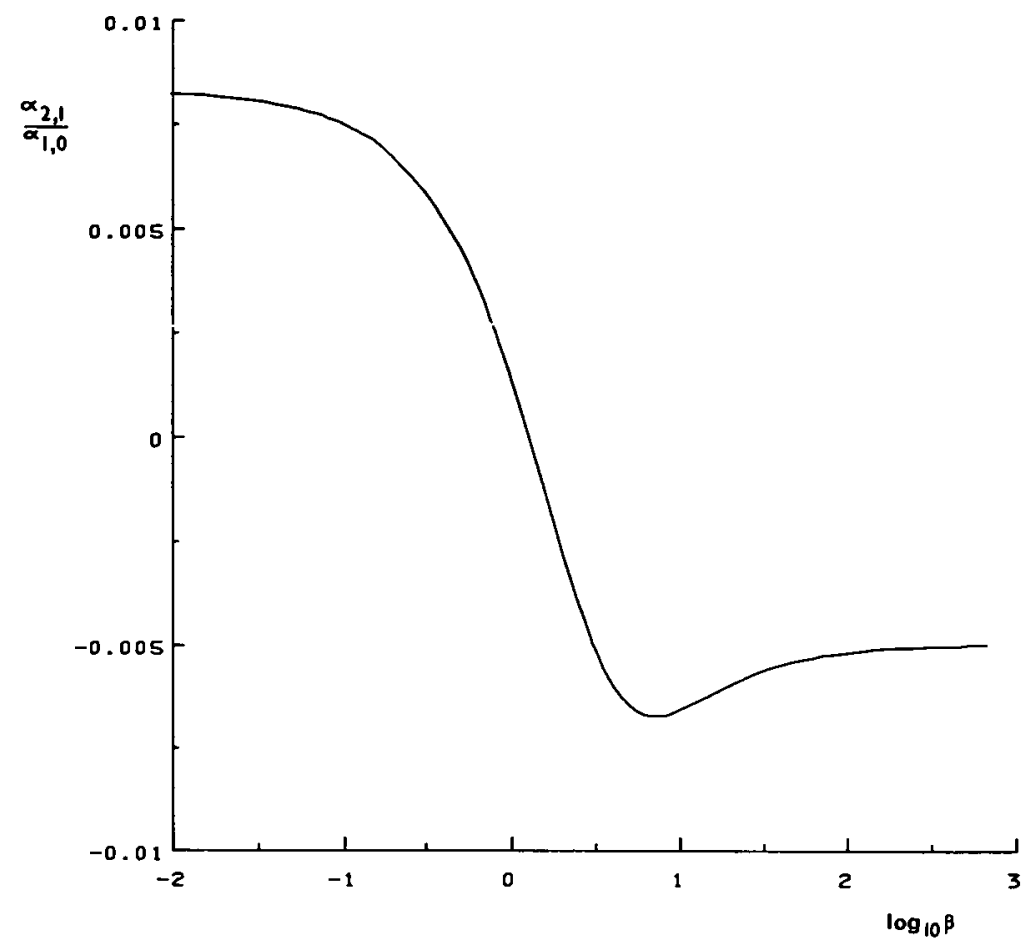

Figure 4. A plot of the skewness parameter $\alpha_{2.1} / \alpha_{1,0}$ defined by (4.14) against the dimensionless wall reaction parameter $\beta$.

The dependence of $\gamma_{1}$ upon $\beta$ is shown in Figure 2. Again, the approximations developed by Sankarasubramanian and Gill, namely

$$
\begin{gathered}
\gamma_{1} \sim 1+\frac{\beta}{3(2+\beta)} \quad \text { for small } \beta, \\
\gamma_{1} \sim \frac{4}{3}\left[1-\frac{1}{\beta}+\frac{1}{j_{0,1}^{2}}\left(1+\frac{2}{\beta}\right)\right] \text { for large } \beta,
\end{gathered}
$$

are displayed; and these may be compared with the improved approximations

$$
\begin{array}{ll}
\gamma_{1} \sim 1+\frac{1}{6} \beta-\frac{1}{48} \beta^{2} & \text { for small } \beta, \\
\gamma_{1} \text { given by }(4.6 \mathrm{~b}, 4.7) & \text { for large } \beta .
\end{array}
$$

The presentation of further results requires the calculation of the function $\phi_{1}$ (or $g_{2}$ ) or, at least, these functions up to an arbitrary multiple of $f_{1}$. The function 
$\phi_{1}$ is given by the summation in (2.12a) with $i=1$, but it is fortunate that the neater form

$$
\begin{aligned}
\phi_{1}=-g_{2}= & \left\{\frac{-4+3 \alpha_{1}^{2}\left(\gamma_{1}-2\right)}{6 \alpha_{1}^{2}}\right\} \rho J_{1}\left(\alpha_{1} \rho\right) \\
& +\frac{1}{3 \alpha_{1}^{2}} \rho^{2} J_{0}\left(\alpha_{1} \rho\right)+\frac{1}{3 \alpha_{1}} \rho^{3} J_{1}\left(\alpha_{1} \rho\right)
\end{aligned}
$$

is also found to be a particular solution of (2.10 a,b). As written, the form (4.9) satisfies neither $\overline{\rho_{1} f_{1}}=0$ nor $\overline{g_{2}}=0$, but arbitrary multiples of $f_{1}$ can be included for these purposes if required. The inclusion or otherwise of such arbitrary multiples does not affect any of the subsequent results.

The dispersion coefficient $\left\{\overline{K f_{1} f_{1}}+P^{2} \overline{\left(u-\gamma_{1}\right) \phi_{1} f_{1}}\right\}$ may now be calculated by performing the summation implied by (2.12a) or by using the form (4.9). The second method is slightly more convenient and, using the reduction formulae given in the Appendix, $D_{e f f}$ is found to be

$$
D_{e f f}=\frac{1}{2} P^{2} M=1+P^{2} \overline{\left(u-\gamma_{1}\right) \phi_{1} f_{1}}
$$

where

$$
\begin{aligned}
\overline{\left(u-\gamma_{1}\right) \phi_{1} f_{1}} & =\frac{4}{45} \alpha_{1}^{-6}\left(\alpha_{1}^{2}+\beta^{2}\right)^{-3} \\
\times\{ & -\alpha_{1}^{10}+\alpha_{1}^{8}\left[-21-14 \beta-3 \beta^{2}\right]+\alpha_{1}^{6}\left[84 \beta+15 \beta^{2}-18 \beta^{3}-3 \beta^{4}\right] \\
& +\left[-84 \beta^{2}+98 \beta^{3}+43 \beta^{4}-4 \beta^{5}-\beta^{6}\right] \\
& \left.+\alpha_{1}^{2}\left[-128 \beta^{4}+34 \beta^{5}+17 \beta^{6}\right]-54 \beta^{6}\right\} .
\end{aligned}
$$

The values of $\overline{\left(u-\gamma_{1}\right) \phi_{1} f_{1}}$ are displayed as a function of $\beta$ in Figure 3 . Included in the figure are the approximations developed by Sankarasubramanian and Gill [15],

$$
\begin{gathered}
\overline{\left(u-\gamma_{1}\right) \phi_{1} f_{1}} \sim \frac{1}{2+\beta}\left\{\frac{1}{4}+\frac{\beta}{45}\right\} \quad \text { for small } \beta, \\
\overline{\left(u-\gamma_{1}\right) \phi_{1} f_{1}} \sim 256\left(1+\frac{6}{\beta}\right) j_{0,1}^{2} \sum_{n=1}^{\infty} \frac{j_{0, n+1}^{2}}{\left(j_{0, n+1}^{2}-j_{0,1}^{2}\right)^{5}} \quad \text { for large } \beta,
\end{gathered}
$$




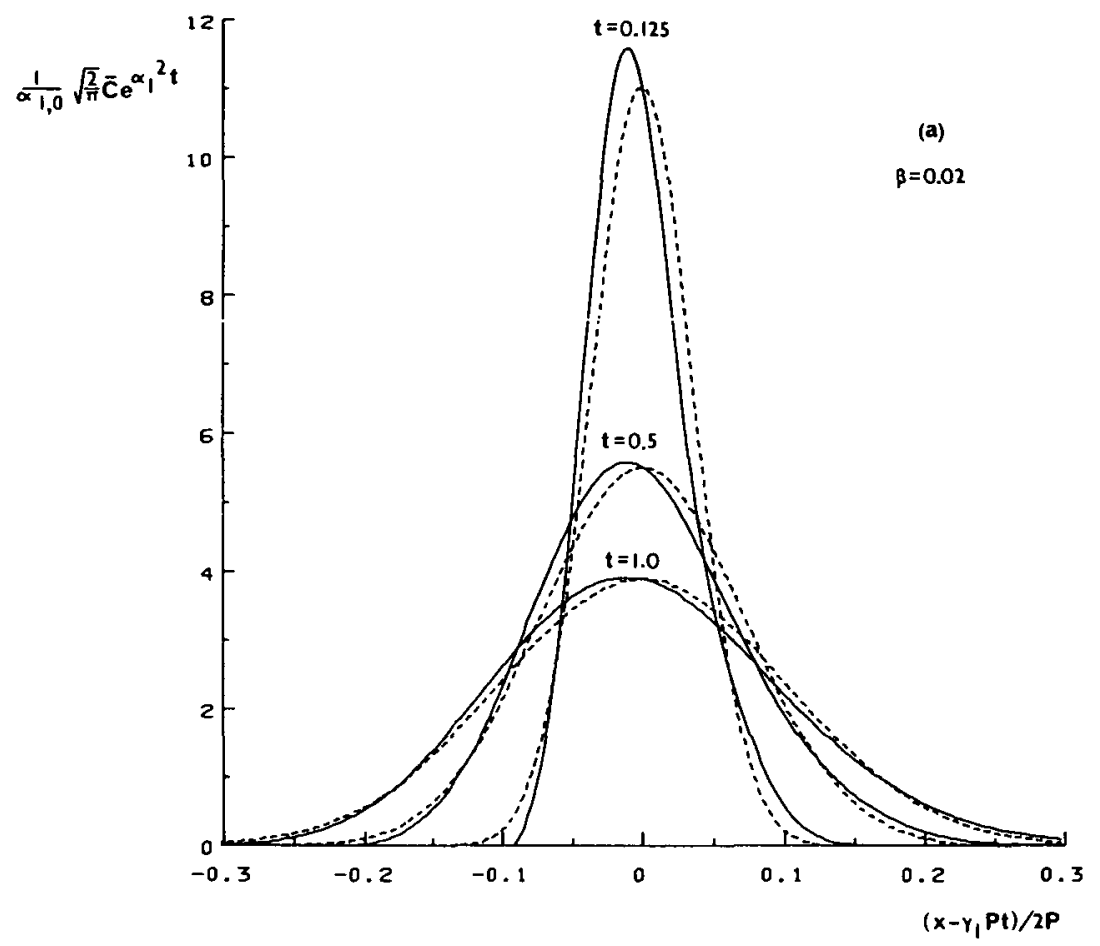

Figure 5. Plots of $\sqrt{2 / \pi} \bar{C} \exp \left\{+\alpha_{1}^{2} t\right\} / \alpha_{1.0}$ as a function of the dimensionless quantity $\left(x-\gamma_{1} P t\right) / 2 P$ at dimensionless times $t=0.125,0.5$ and 1.0 : plot; case (a) is for $\beta=0.02$ and case (b) for $\beta=50$. , two term plot; -----, one term (Gaussian)

and the improved approximations

$$
\begin{gathered}
\overline{\left(u-\gamma_{1}\right) \phi_{1} f_{1}} \sim \frac{1}{48}+\frac{1}{360} \beta-\frac{37}{4320} \beta^{2} \text { for small } \beta, \\
\overline{\left(u-\gamma_{1}\right) \phi_{1} f_{1}} \text { given by }(4.6 \mathrm{~b}, 4.11) \text { for large } \beta .
\end{gathered}
$$

As a further application of the theory developed in Sections 2 and 3, we now examine the parameter $\alpha_{2,1}$ defined by (3.20). This parameter is of some importance as it determines the skewness of the asymptotic distribution (see 3.24), and it is required for a two-term expression for $C$ (see $3.3,3.17,3.18$ ). Omitting a large amount of algebra, $\alpha_{2,1} / \alpha_{1,0}$ is eventually found to be

$$
\frac{\alpha_{2,1}}{\alpha_{1,0}}=\frac{1}{M} \sum_{j \neq 1}\left\{\frac{1}{\mu_{1}-\mu_{j}} \sum_{k \neq 1} \frac{\overline{u f_{1} f_{k}} \overline{\left(u-\gamma_{1}\right) f_{k} f_{J}}}{\mu_{1}-\mu_{k}}\right\} \overline{u f_{1} f_{j}}
$$

where $M$ and $\gamma_{1}$ are given by (4.10) and (4.7), and the various cross-sectional means in the expressions may be evaluated using standard integrals. The quantity 


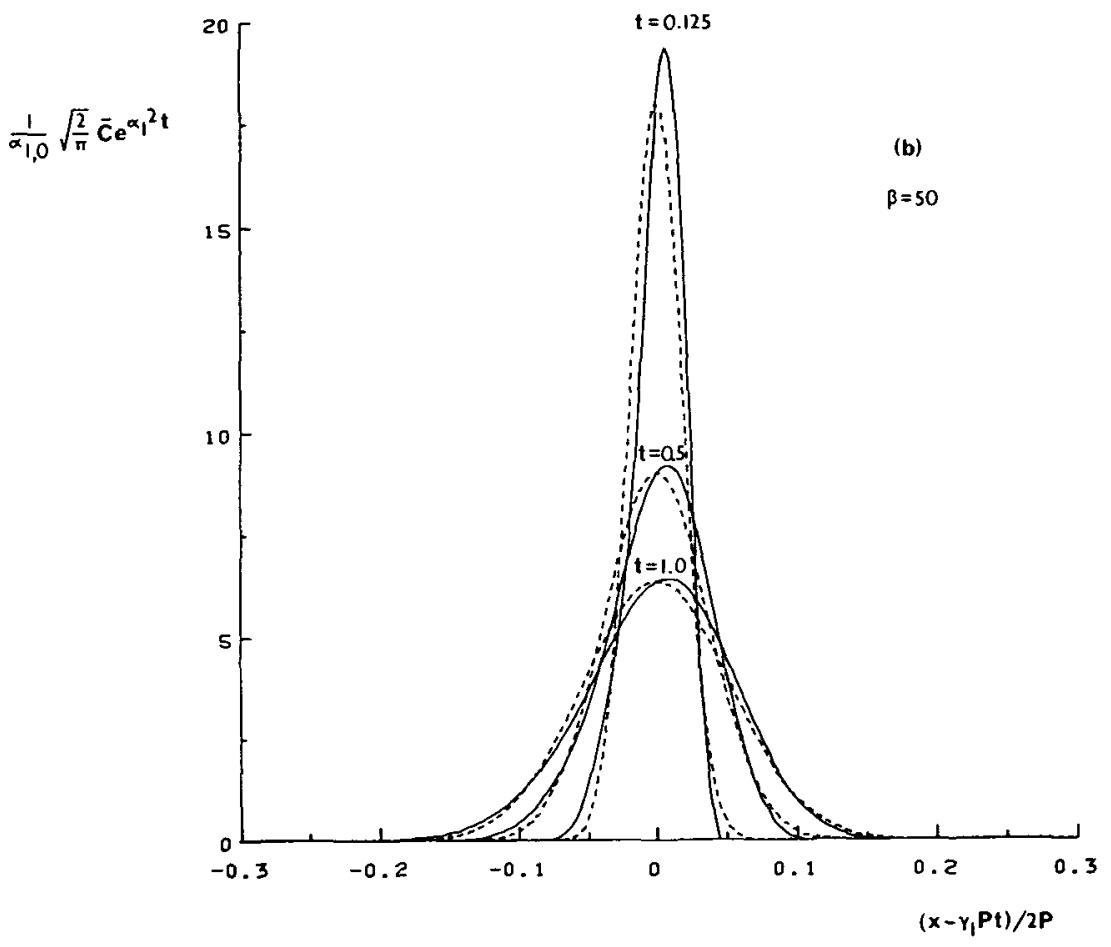

Figure 5. (continued)

$\alpha_{2,1} / \alpha_{1,0}$ is shown in the high Péclet number limit (that is neglecting the constant 1 on the right hand side of 4.10) in Figure 4.

A physical interpretation of the information contained in Figures 1 to 3 has been given by Sankarasubramanian and Gill. The plot in Figure 4 is new, however, and is most interesting as it shows a change in the sign of the skewness of the distribution for $\beta$ slightly greater than 1. Thus, for $\beta$ near 1, the Gaussian approximation to $C$ would be unexpectedly good compared with the approximation described by Chatwin for $\beta=0$. As $\beta \rightarrow 0, \alpha_{2,1} / \alpha_{1,0}$ agrees well with the theoretical value of $1 / 120$ [5, equation 4.6] whilst, as $\beta \rightarrow \infty, \alpha_{2,1} / \alpha_{1.0}$ approaches $-1 / 200$.

To conclude this section, we present plots for one and two terms of the asymptotic series

$$
\bar{C}(x, t)=\alpha_{1,0} \bar{f}_{1} e^{-\alpha_{1}^{2} t}\left\{\frac{1}{T}+\frac{\alpha_{2,1}}{\alpha_{1,0}} \frac{H_{3}(X)}{T^{2}}\right\} \exp \left\{-\frac{1}{2} X^{2}\right\}
$$

in various ways so as to deduce when $\bar{C}(x, t)$ can be described by the one term Gaussian approximation. All of the variables mentioned in (4.15) have been 
described in this section, and the plots are again for the high Péclet number limit in which $M$ becomes $\overline{\left(u-\gamma_{1}\right) \phi_{1} f_{1}}$. The variables $X$ and $T$ are

$$
X=\left(x-\gamma_{1} P t\right) / P T, \quad T=(M t)^{1 / 2}
$$

and $\alpha_{2,1} / \alpha_{1,0}$ is given by (4.14).

Figures 5a, b show $\sqrt{2 / \pi} \bar{C} e^{\alpha_{1}^{2} t}$ as a function of $\left(x-\gamma_{1} P t\right) / 2 P$ at $t=0.125$, 0.5 and 1.0 and for $\beta=0.02$ and 50.0. The scales are chosen to enable a direct comparison with the one and three term plots of Chatwin [5, Figure 3]. For $\beta=0.02$, the similarity of the present results to Chatwin's is clear: the peak of $\bar{C}$ occurs behind the centre of mass and the asymmetry in the two term curve is practically negligible for $t$ at 1.0. For $\beta=50.0$, the peak of $\bar{C}$ occurs ahead of the centre of mass, and, for practical purposes again, there appears to be no significant different between the 1 and 2 term approximations for $t$ greater than 1 . Plots of (4.15) for $\beta=1,0$ were prepared for $t=0.125,0.5,1.0$, but are not included here as they are almost indistinguishable from the Gaussian curve by virtue of the change of sign of $\alpha_{2,1}$ near 1 .

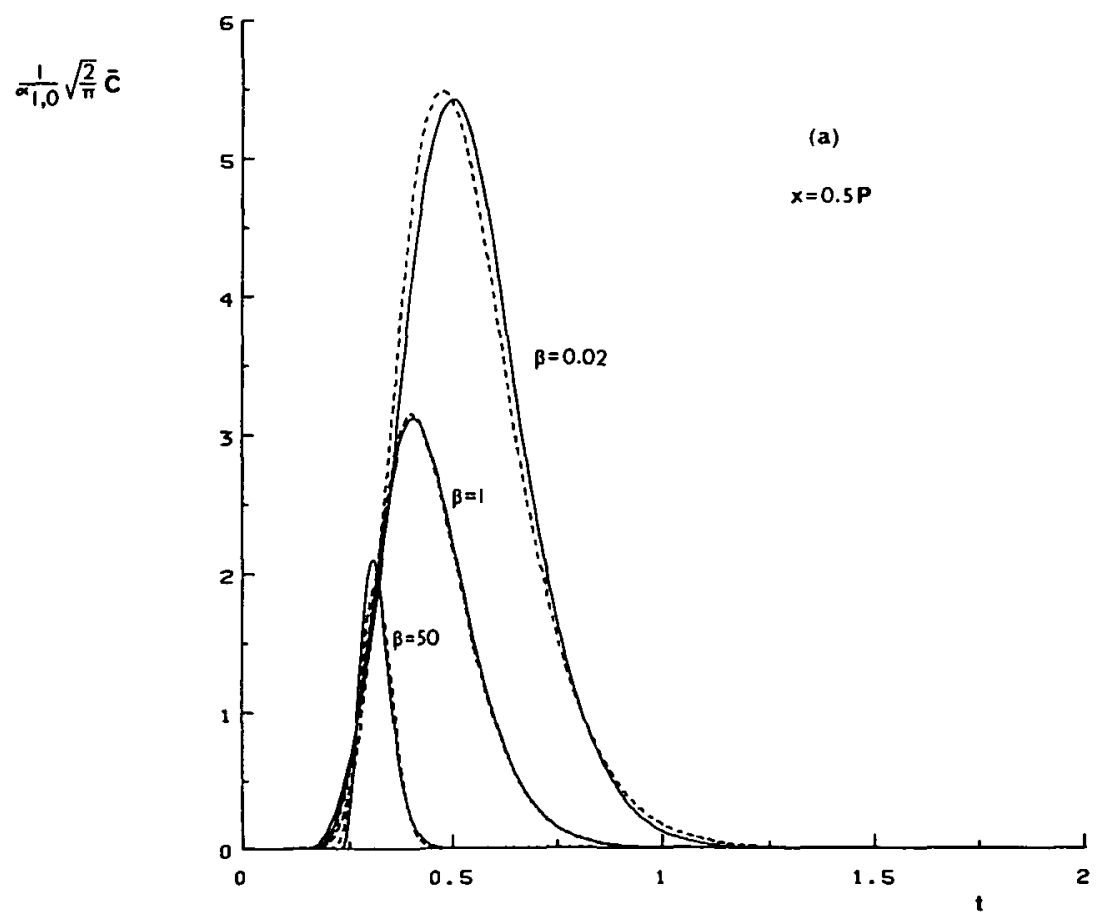

Figure 6. Plots of $\sqrt{2 / \pi} \bar{C} / \alpha_{1.0}$ as a function of the dimensionless time $t$ for $\beta=0.02,1,50$ : two term plot; -...., one term (Gaussian) plot; cases $(a, b, c)$ are at dimensionless distances $(0.5 P, 1.0 P, 1.5 P)$ downstream of the injection point. 

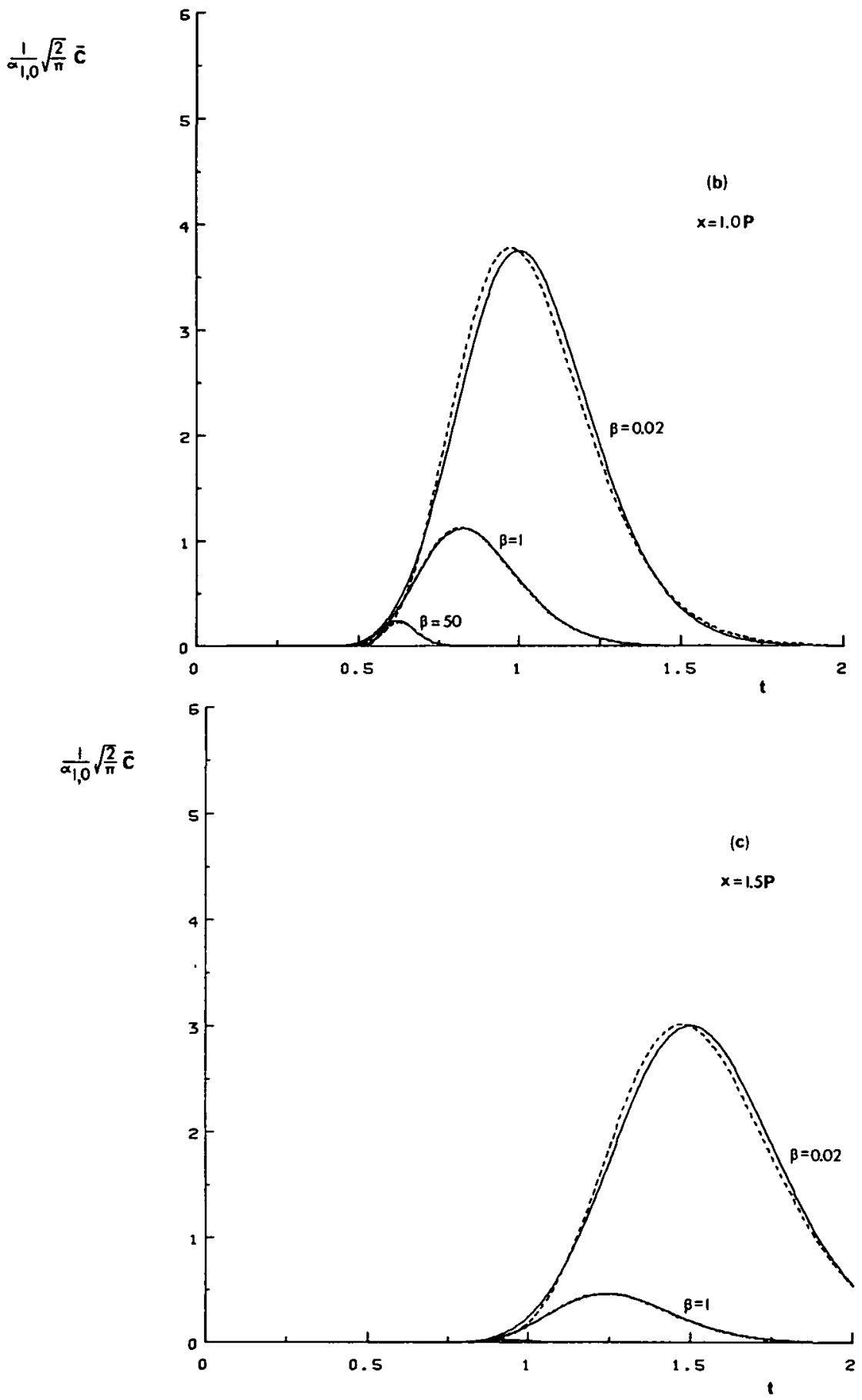

Figure 6. (continued) 
Plots of $\bar{C}$ against $x$ for fixed values of the time represent a 'photograph' of the distribution, and are quite different from the usual measurement which is of $\bar{C}$ against $t$ at fixed values of $x$. Accordingly Figures $6 \mathrm{a}, \mathrm{b}, \mathrm{c}$ display $\sqrt{2 / \pi} \bar{C} / \alpha_{1,0}$ against $t$ for $\beta=0.02,1,50.0$ at $x=0.5 P, 1.0 P, 1.5 P$. Some noteworthy points are: the similarity of the one and two term approximations is evident for $\beta=1.0$, even for $t=0.5$; the $\beta=50.0$ curves are almost indistinguishable from zero at $x=1.5 P$; the change in skewness of the curves for small and large $\beta$; and the closeness of the one and two term approximations for all $\beta$ for $t>1.0$.

\section{Conclusion}

A direct asymptotic theory has been developed to describe the dispersion of reactive contaminants injected into parallel flow. The theory is somewhat less general than references $[6,7,15]$ based on Taylor-Gill expansions, but gives comparable results at large times and is arguably easier to apply. The theory has been applied in detail to the flow reactor example, and confirms the results of reference [15] for the total amount of solute, the speed of the centre of mass, and the dispersion coefficient. The small $\beta$ and large $\beta$ expansions developed herein are superior to those in reference [15]. Also, the skewness parameter $\alpha_{2,1} / \alpha_{1,0}$ has been presented as a function of $\beta$ for the first time: as $\beta \rightarrow 0$, this parameter agrees with Chatwin's result [5], and it changes sign for $\beta$ slightly greater than 1 .

One and two term asymptotic expansions for $\bar{C}(x, t)$ have been plotted for various values of $x, t$ and $\beta$ in the flow reactor example. The broad conclusion is that these expansions are identical for practical purposes for non-dimensional times $t$ greater than 1 , although, for $\beta$ near 1 , the change in skewness means that the one and two term expansions are practically identical for much smaller $t$. It is noted that the shape of the original injected cloud affects the asymptotic expansion at the third and subsequent terms. Sufficient details are presented to compute the third term if required, but the calculation depends in a complicated manner on $\beta, P$ and the shape of the initial cloud and is not included here. Finally, the present work has recently been used to determine diffusion coefficients for mixtures of gases at low pressures [14]. The shape of the initial distribution in this application affected the distribution observed further down the tube, but was accounted for by partitioning the initial distribution into a succession of short input pulses. By such a device, the importance of the third and subsequent term in the series can be diminished. 


\section{Acknowledgement}

The author would like to thank Dr. A. N. Stokes for several perceptive comments made on a preliminary draft of this work.

\section{Appendix}

The following reduction formulae were used in the evaluation of $\overline{\left(u-\gamma_{1}\right) \phi_{1} f_{1}}$ in Section 4:

$$
\begin{gathered}
\int z^{2 n} J_{1}(z) J_{0}(z) d z=-\frac{1}{2} z^{2 n} J_{0}^{2}(z)+n \int z^{2 n-1} J_{0}^{2}(z) d z \\
(n+2) \int z^{n+2} J_{0}^{2}(z) d z=-\frac{1}{4}(n+1)^{3} \int z^{n} J_{0}^{2}(z) d z \\
+\frac{1}{2} z^{n+1}\left[\left\{z J_{0}^{\prime}(Z)-\frac{1}{2}(n+1) J_{0}(z)\right\}^{2}+\left\{z^{2}+\frac{1}{4}(n+1)^{2}\right\} J_{0}^{2}(z)\right] .
\end{gathered}
$$

(The second formula is a special case of Schafheitlin's reduction formula.)

\section{References}

[1] R. Aris, "On the dispersion of a solute in a flud flowing through a tube," Proc. Roy. Soc. London Ser. A 235 (1956), 67-77.

[2] R. Aris, "On the dispersion of a solute by diffusion, convection and exchange between phases," Proc. Roy. Soc. London Ser. A 252 (1959), 538-550.

[3] N. G. Barton, "On the method of moments for solute dispersion," J. Fluid Mech. 126 (1983), 205-218.

[4] R. L. Brown, "Tubular flow reactors with first-order kinetics," J. Res. Nat. Bur. Standards 83 (1978), 1-8.

[5] P. C. Chatwin, "The approach to normality of the concentration distribution of a solute in a solvent flowing along a straight pipe," J. Fluid Mech. 43 (1970), 321-352.

[6] A. E. de Gance and L. E. Johns, "The theory of dispersion of chemically active solutes in a rectilinear flow field," Appl. Scl. Res. 34 (1978), 189-225.

[7] A. E. de Gance and L. E. Johns, "On the dispersion coefficients for Poiseuille flow in a circular cylinder," Appl. Sci. Res. 34 (1978), 227-258.

[8] P. G. Dickens, D. Schofield and J. Walsh, "Recombination of atoms at surfaces. Part 8. The three-dimensional diffusion equation," Trans. Faraday Soc. 56 (1960) 225-233.

[9] H. B. Fischer, J. Imberger, E. J. List, R. C. Y. Koh and N. H. Brooks, Mixıng in inland and coastal waters (Academic, New York, 1979).

[10] W. M. Gill and R. Sankarasubramanian, "Exact analysis of unsteady convective diffusion," Proc. Roy. Soc. London Ser. A 316 (1970), 341-350.

[11] C. J. Howard, "Kinetic measurements using flow tubes," J. Phys. Chem. 83 (1976), 3-8.

[12] T. R. Marrero and E. A. Mason, "Gaseous diffusion coefficients," J. Phys. Chem. Ref. Data I (1972), 3-118.

[13] J. C. Pirkle and V. G. Sigillito, "Variational treatment of chemical reaction and diffusion in a catalytic tubular reactor," Internat. J. Engrg. Sci. 10 (1972), 553-559. 
[14] I. C. Plumb, K. R. Ryan and N. G. Barton, "A method for the measurements of diffusion coefficients of labile gas phase species: the diffusion coefficient of $O\left({ }^{3} \mathrm{P}\right)$ in $\mathrm{He}$ at $294 \mathrm{~K}$," Internat. J. Chem. Kinetics 30 (1983).

[15] R. Sankarasubramanian and W. N. Gill, "Unsteady convective diffusion with interphase mass transfer," Proc. Roy. Soc. London Ser. A 333 (1973), 115-132. Corrected in Proc. Roy. Soc. London Ser. A 341 (1974), 407-408.

[16] R. Smith, "The early stages of contaminant dispersion in shear flow," J. Fluid Mech. III (1981), 107-122.

[17] R. Smith, "A delay-diffusion description for contaminant dispersion," I. Flujd Mech. !05 (1981), 469-486.

[18] G. I. Taylor, "Dispersion of soluble matter in solvent flowing slowly through a tube," Proc. Roy. Soc. London Ser. A 219 (1953), 186-203.

[19] G. 1. Taylor, "The dispersion of matter in turbulent flow through a pipe," Proc. Roy. Soc. London Ser. A 223 (1954), 446-468.

[20] R. E. Walker, "Chemical reaction and diffusion in a catalytic tubular reactor," Phys. Fluids 4 (1961), 1211-1216. 